\title{
REALIZATION OF DIGITAL FILTERS WITH COMPLEX COEFFICIENTS
}

\author{
$U D C(621.372 .852 .1: 512.732)$
}

\section{Saša Nikolić, Goran Stančić, Stevica Cvetković}

University of Niš, Faculty of Electronic Engineering, Republic of Serbia

\begin{abstract}
A comprehensive analysis of realization of digital filters with complex coefficients obtained by decomposition of real digital filters, using complex and real allpass networks, is given in the paper. Realization of complex coefficient filters obtained directly in the $z$ domain is also discussed in the paper. An analysis of hardware costs for different structures used for realization of complex coefficient filters is presented.
\end{abstract}

Key words: Digital filters, all-pass filters, filters with complex coefficients, filter realization

\section{INTRODUCTION}

Digital filters with complex coefficients have been very attractive last several years because of many advantages for processing of digital signals. Transfer functions of these filters are complex functions and input signals must be separated to real and imaginary parts, in order to be represented as complex signals. Complex filtering is very popular in telecommunications where complex representation of signals allows simple realization and interpretation of complex tasks such as sampling, quantization or modulation.

The theory of complex filters has been well known for a long time, but in the last two decades the work on realization of these filters is much more actualized $[5,9,10,11,12]$. Today, complex filters are an important part of modern telecommunications. Speech processing and adaptive filtering cannot be imagined without using the digital filters with complex coefficients.

There are several different structures for realization of complex digital filters. The problem is very topical because there is a need to use a smaller number of components in order to realize a digital filter. The main objective of this paper is to investigate the realization of complex filters using the allpass complex sections. In this paper we also investigated the hardware cost for realization of complex digital filters based on

Received February 24, 2018

Corresponding author: Saša V. Nikolić

Faculty of Electronic Engineering, Aleksandra Medvedeva 14, 18000 Niš, Republic of Serbia

E-mail: sasa.nikolic@elfak.ni.ac.rs 
decomposition of a filter with real coefficients of order $2 \mathrm{~N}$ on a filter with complex coefficients of order $\mathrm{N}$. We discussed the direct realization, canonic realization, cascade and parallel realization of a digital filter with complex coefficients. Comparing different structures we calculated the number of necessary components for every realization.

Structures for realization of filters with real coefficients are very well known in existing literature $[1,2,3,4]$. The numbers of required components for different structures for realization of these filters are displayed in Table 1 . It is obvious that for all structures the number of required components is the same excluding the direct realization.

Table 1 Hardware cost for realization of filters with real coefficients of order $\mathrm{N}$.

\begin{tabular}{lccc}
\hline Structure & Delay lines & Multiplicators & Adders \\
\hline Direct realization & $2 \mathrm{~N}$ & $2 \mathrm{~N}+1$ & $2 \mathrm{~N}$ \\
Canonic realization & $\mathrm{N}$ & $2 \mathrm{~N}+1$ & $2 \mathrm{~N}$ \\
Cascade realization & $\mathrm{N}$ & $2 \mathrm{~N}+1$ & $2 \mathrm{~N}$ \\
Parallel realization & $\mathrm{N}$ & $2 \mathrm{~N}+1$ & $2 \mathrm{~N}$ \\
\hline
\end{tabular}

Some types of filters (for example Butterworth, Chebyshev, Elliptic ...) with real coefficients can be realized using the allpass networks [5]. In the case when the order of filter with real coefficients $\mathrm{N}$ is odd this filter can be realized using two real allpass sections where their order differs for one (the allpass filter orders will be $(\mathrm{N}-1) / 2$ and $(\mathrm{N}+1) / 2)[13,14,15,16]$.

If the order of filter with real coefficients $\mathrm{N}$ is even this filter can be realized with two allpass sections with complex coefficients of the same order N/2. At the first moment, taking into account that realization of a complex multiplier requires four real multipliers, one can conclude that realization using complex sections is more complex. However coefficients of two complex sections are conjugate-complex to each other and for this reason it is enough to realize only one complex section of order N/2. The real part of the output signal and imaginary part of the output signal corresponds to mutually complementary filters outputs.

\section{COMPLEX SignALS}

Complex signal processing allows simple interpretation of complicated processing tasks, such as modulation, sampling and quantization. Digital filters with complex coefficients have real and imaginary inputs and outputs and signals they process have to be separated into real and imaginary parts in order to be represented as complex signals. Digital complex filters have many areas of application. The most interesting implementation of digital complex filters is realization of SSB (Single Side band) transmitters and receivers. Complex filtering is also preferred when DFT is carried out, as it is linear combination of complex components.

The complex signal known as an analytic signal consists of an input signal $x[n]$ as his real part while the imaginary part of the analytic signal is obtained by the Hilbert transformer applied on the input signal $x[n]$, as given in Figure 1. Frequency components of real and imaginary parts of the analytic signal have $\pi / 2$ phase difference at all frequencies. As a consequence, the analytic signal has nonzero spectral components only 
at positive frequencies. Such signal is convenient for modulation giving an SSB output signal. In a given frequency band the number of communication channels is doubled due to the narrower spectrum of the analytic signal.

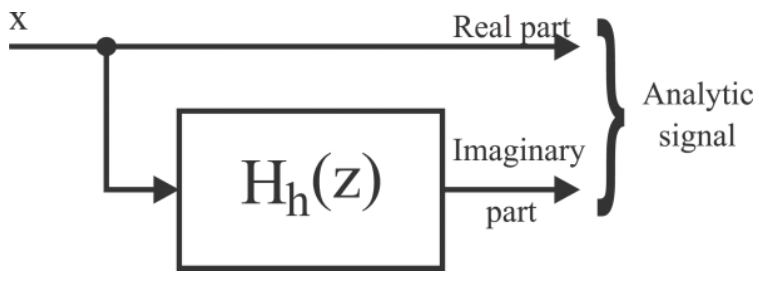

Fig. 1 Realization of analytic signal.

\section{REALISATION USING ALLPASS SECTIONS}

Besides the standard structures for filter realization (direct, cascade and parallel), with the advent of the filter banks, parallel connections of the allpass filters become a very attractive solution taking into account the fact that with the realization of one filter it is possible to realize a complementary filter using only one additional adder. This configuration demonstrates the true benefits in the cases where it is necessary to implement two complementary filters. Therefore, it is common in the existing literature when displaying hardware costs to count the necessary number of multipliers, adders and delay line per one transfer function (i.e., the total number of components is divided by two). In the case when filters are implemented by the standard approach it is necessary to completely independently implement both filters (for example lowpass and highpass filters). If one insists on the linear phase it is necessary to add in the cascade appropriate phase correctors. The hardware cost for realization of linear phase filter using the allpass networks is lower comparing with the standard realization with phase correctors [13].

The structure shown in the Fig. 2 is characterized by a straightforward connection between the amplitude and phase characteristics of all the allpass filters from parallel branches.

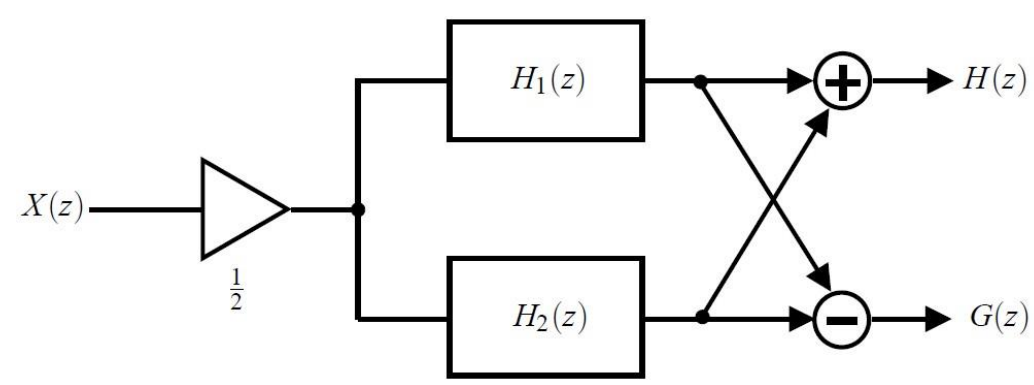

Fig. 2 Parallel connection of two allpass filters. 
Transfer functions $H(z)$ and $G(z)$, amplitude characteristics $\left|H\left(e^{j \theta}\right)\right|$ and $\left|G\left(e^{j \theta}\right)\right|$ and phase of the resulting filters $\psi_{H}(\theta)$ and $\psi_{G}(\theta)$ are given with the next formulas

$$
\begin{aligned}
& H(z)=\frac{H_{1}(z)+H_{2}(z)}{2},\left|H\left(e^{j \theta}\right)\right|=\left|\cos \left(\frac{\varphi_{1}(\theta)-\varphi_{2}(\theta)}{2}\right)\right|, \psi_{H}(\theta)=\frac{\varphi_{1}(\theta)+\varphi_{2}(\theta)}{2}, \\
& G(z)=\frac{H_{1}(z)-H_{2}(z)}{2},\left|G\left(e^{j \theta}\right)\right|=\left|\sin \left(\frac{\varphi_{1}(\theta)-\varphi_{2}(\theta)}{2}\right)\right|, \psi_{G}(\theta)=\frac{\varphi_{1}(\theta)+\varphi_{2}(\theta)-\pi}{2},
\end{aligned}
$$

Based on the above expressions we can conclude that these filters do not need phase correctors, because it is obvious that the design boils down to the problem of phase approximation while simultaneously satisfies the conditions set by the amplitude of the resulting filter. Another advantage of the structure refers to the case of designing a filter with an approximately linear phase in which case one of the allpass filters, becomes a pure delay line, i.e. it does not contain adders and multipliers, in other words it is necessary to design only one IIR allpass filter whose phase will approximate an ideal piecewise linear phase in all the passbands and stopbands. Taking into account the fact that the delay line phase is ideal linear, overall phase approximation error is due to the allpass filter design. Hence, an equripple approximation of the allpass filter phase will give elliptic magnitude characteristics.

Phase characteristics of allpass filters are displayed in Fig. 3. According to equation (1) passbands of the selective filter $H(z)$ will be realized at frequencies where phase difference is approximately equal to zero while stopbands would be located at frequencies where phase difference is approximately equal to $\pi \mathrm{rad}$. At Fig. 3. functions $\Phi_{2}(\theta)$ denotes linear phase of delay line and $\Phi_{1}(\theta)$ corresponds to the IIR allpass phase. It is clear that cutoff frequency will be located at $\theta_{g}$ where phase of the allpass filter has $\pi$ rad phase jump.

With regard to the expression (1), we see that the next formula is valid

$$
\left|H\left(e^{j \theta}\right)\right|^{2}+\left|G\left(e^{j \theta}\right)\right|^{2}=1
$$

and characteristics of the filter in the passband are directly related to the characteristics of the complementary filter in the stopband. This connection is displayed in Fig. 4. If one takes a logarithmic scale on the $\mathrm{x}$ axis, this relationship is linear which allows us to easily come up with a formula that describes this relationship and it is given by the equation

$$
A_{\max L P}=10^{k A_{\min H P}+c},
$$

where constants are $k=-0.10003362$ and $c=0.640352064$. Maximal attenuation in the passband is marked with $A_{\max L P}$ and minimal attenuation in the stopband is marked with $A_{\operatorname{minHP}}$. 


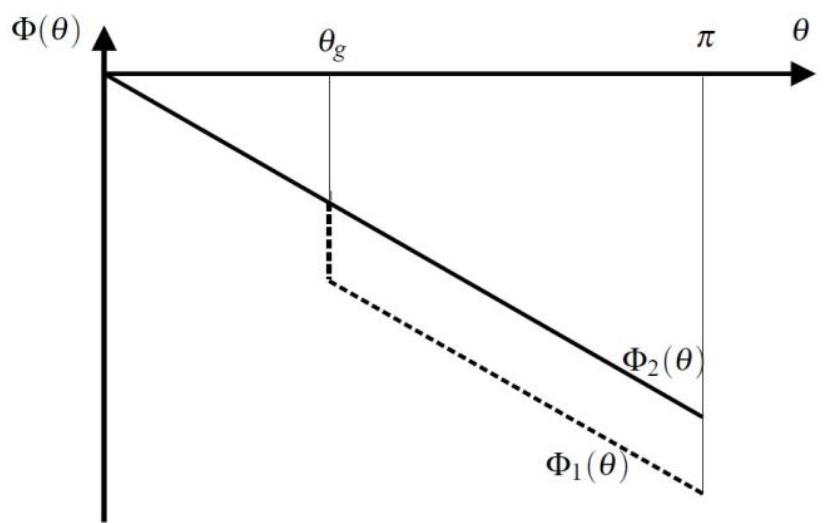

Fig. 3 Illustration of allpass filters phase characteristics.

Considering the equation (1) it is easy to understand why the problem of designing prescribed magnitude characteristics is reduced to the allpass phase approximation problem. From Fig. 4. one can observe that if a stopband attenuation higher than $30 \mathrm{~dB}$ is achieved, the complementary filter has a very small passband attenuation. That is the reason to use as input parameters only the stopband attenuations of complementary filters. Such an approach will provide solution with maximal passband attenuation far below $1 \mathrm{~dB}$.
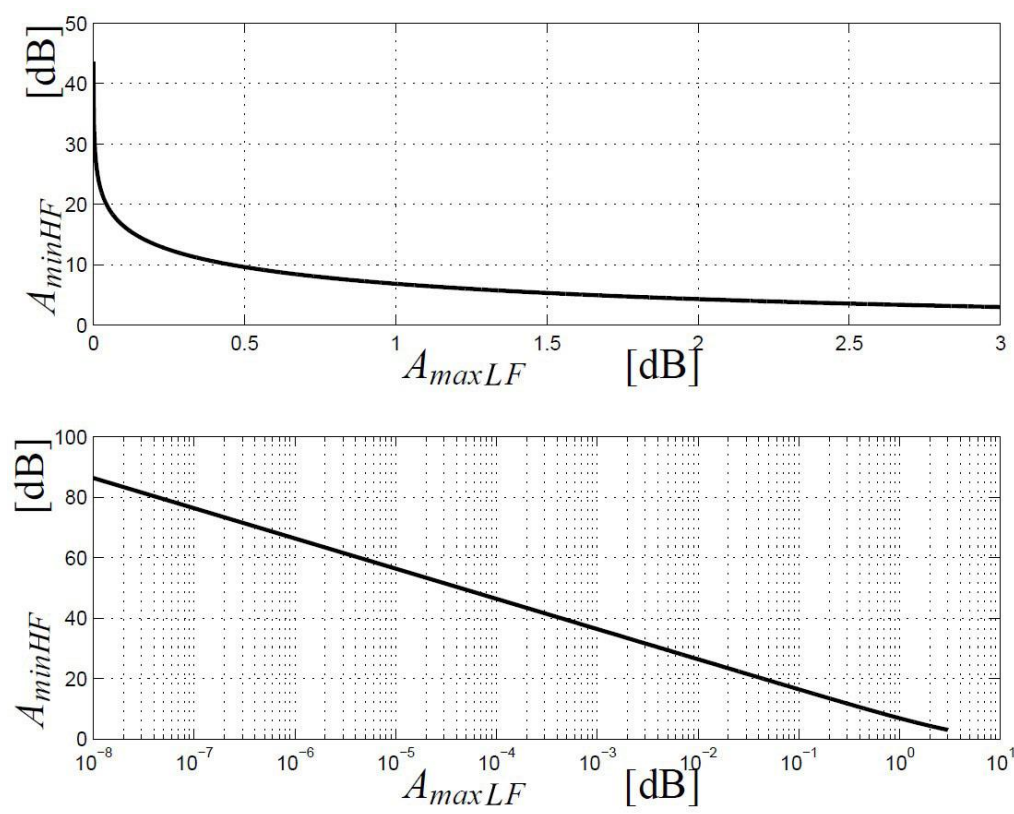

Fig. 4 Dependency between the minimal attenuation of the highpass filter in the stopband $A_{\operatorname{minHP}}$ and maximal attenuation of the lowpass filter in the passband $A_{\max L P}$. 
In order to illustrate the importance of equation (4) we realized a Chebyshev lowpass filter of eighth order. We made also decomposition of this filter on two complex allpass sections of order four. The coefficients of the eighth order Chebyshev digital lowpass filter with cutoff frequency $0.45 \pi$ with maximal attenuation in the passband $A_{\max }=3 \mathrm{~dB}$ are displayed in Table 2 together with the coefficients of the transfer functions of the corresponding complex allpass filters of the fourth order.

Table 2 The coefficients of the Chebyshev filter and complex allpass filters obtained by decomposition (coefficients of polynomial numerator $b_{i}$ and denumerator $a_{i}$, Chebyshev filter of eighth order, cutoff frequency $\theta_{p}=0.4 \pi$ and coefficients at numerator of the complex allpass filter $\mathrm{H}_{1}(\mathrm{z})$ are $\left(a_{1 i}\right)$ and complex allpass filter $H_{2}(z)$ are $\left(a_{2 i}\right)$.

\begin{tabular}{ccrrr}
\hline$i$ & $b_{i}$ & \multicolumn{1}{c}{$a_{i}$} & \multicolumn{1}{c}{$a_{1 i}$} & \multicolumn{1}{c}{$a_{2 i}$} \\
\hline 1 & 0.0001745 & & 0.5622295 & 0.5622295 \\
& & & $-\mathrm{j} 0.4184267$ & $+\mathrm{j} 0.4184267$ \\
2 & 0.0013963 & -4.4082892 & -1.7392394 & -1.7392394 \\
& & & $+\mathrm{j} 0.8796079$ & $-\mathrm{j} 0.8796079$ \\
3 & 0.0048871 & 10.3501526 & 2.6317070 & 2.6317070 \\
& & & $-\mathrm{j} 0.9207915$ & $+\mathrm{j} 0.9207915$ \\
4 & 0.0097742 & -15.9600831 & -2.2041447 & -2.2041447 \\
& & & $+\mathrm{j} 0.4780012$ & $-\mathrm{j} 0.4780012$ \\
5 & 0.0122177 & 17.4061751 & 1 & 1 \\
6 & 0.0097742 & -13.6526950 & & \\
7 & 0.0048871 & 7.5284778 & & \\
8 & 0.0013963 & -2.6918063 & & \\
9 & 0.0001745 & 0.4911829 & & \\
\hline
\end{tabular}

We can conclude that one of the poles, from the complex conjugate pairs of the starting filter function, belonging to one or the other all pass network. Here is easy to see why the basic building block for the realization is a complex section of the first order. It is clear that a selected filter has a relatively high attenuation in the passband, which corresponds to a higher phase error and from this reason complementary filter will be practically unusable.

Pole positions of the Chebyshev filter and pole positions of both complex allpass sections are displayed in Fig. 5 together with attenuation of the lowpass filter and his complementary highpass filter. It is obviously from Fig. 5 that the complementary highpass filter is unusable because the minimal attenuation in the stopband of the complementary highpass filter is less than $5 \mathrm{~dB}$. It is a consequence of a bad choice of the maximal passband attenuation of the lowpass filter. In order to get a complementary highpass filter with minimal attenuation in the stopband more than $40 \mathrm{~dB}$, using Fig. 4, it can be seen that the correct choice for maximal attenuation in the passband of the lowpass filter is about $0.0004 \mathrm{~dB}$. Using this value for maximal attenuation in the passband of the lowpass filter will yield a complementary highpass filter with satisfied characteristics. 

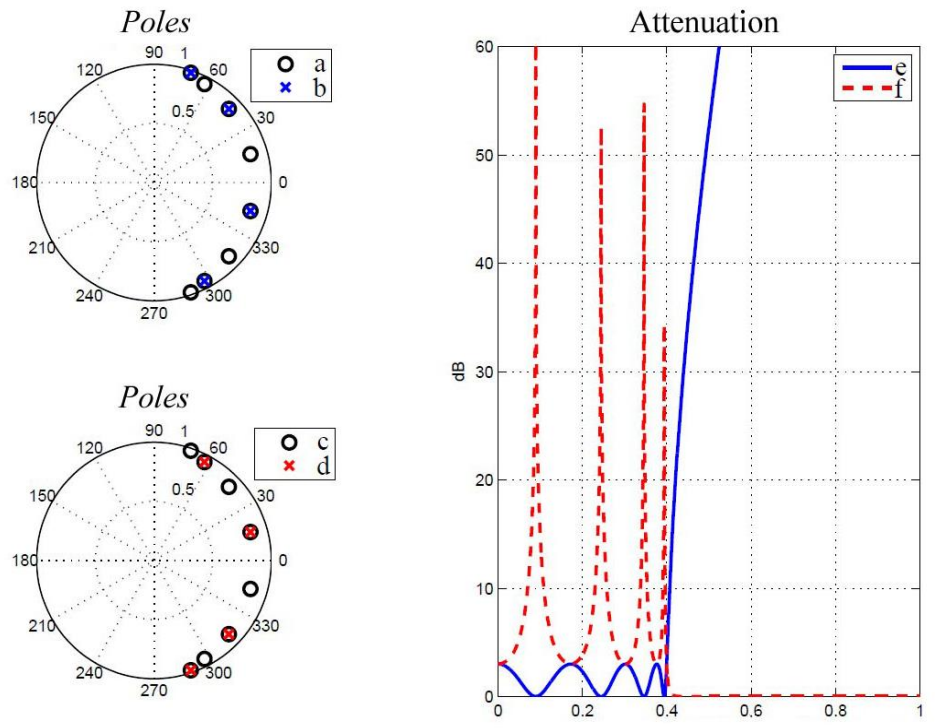

Fig. 5 Pole positions of Chebyshev filter and both all pass sections and attenuation characteristics of Chebyshev and its complementary filter.

\section{STRUCTURES FOR REALIZATION OF COMPLEX FILTERS}

A Filter with complex coefficients can be obtained besides even order real coefficients transfer function decomposition also using design directly in the $\mathrm{z}$ domain. For realization of these filters it is possible to use standard structures for realization which will be discussed in this chapter.

\subsection{Direct realization of digital filters with complex coefficients}

Transfer function of the filter with complex coefficients realized in direct form is given with

$$
H(z)=\frac{Y(z)}{X(z)}=\frac{\sum_{k=0}^{M}\left(b_{k r}+b_{k i}\right) z^{-k}}{1+\sum_{k=1}^{M}\left(a_{k r}+a_{k i}\right) z^{-k}} .
$$

This expression can be rewritten in the more convenient form

$$
\frac{Y(z)}{X(z)}=\frac{\left(b_{0 r}+b_{0 i}\right)+\left(b_{1 r}+b_{1 i}\right) z^{-1}+\ldots+\left(b_{M r}+b_{M i}\right) z^{-M}}{1+\left(a_{1 r}+a_{1 i}\right) z^{-1}+\ldots+\left(a_{M r}+a_{M i}\right) z^{-M}} .
$$

Using the inverse $Z$ transform we can calculate real and imaginary parts of the output of a digital filter as 
and

$$
\begin{aligned}
y_{r}(n)= & b_{0 r} x_{r}(n)+b_{1 r} x_{r}(n-1)+\ldots+b_{M r} x_{r}(n-M) \\
& -a_{1 r} y_{r}(n-1)+a_{1 i} y_{i}(n-1)-\ldots \\
& -a_{M r} y_{r}(n-M)+a_{M i} y_{i}(n-M)
\end{aligned}
$$

$$
\begin{aligned}
y_{i}(n)= & b_{0 i} x_{r}(n)+b_{1 i} x_{r}(n-1)+\ldots+b_{M i} x_{r}(n-M) \\
& -a_{1 i} y_{r}(n-1)-a_{1 r} y_{i}(n-1)-\ldots \\
& -a_{M i} y_{r}(n-M)-a_{M r} y_{i}(n-M)
\end{aligned}
$$

The direct realization of the filter with complex coefficients is displayed in Fig. 6. From this figure we can count a required number of components for this structure. The number of required multipliers is $6 M+2$, of required adders is $6 M$ and of delay lines is $3 M$. These numbers of components are necessary for realization of two complementary transfer functions. It means that for a direct realization of a filter of order $N / 2$ we need $3 N / 2+1$ multipliers, $3 N / 2$ adders and $3 N / 4$ delay lines.

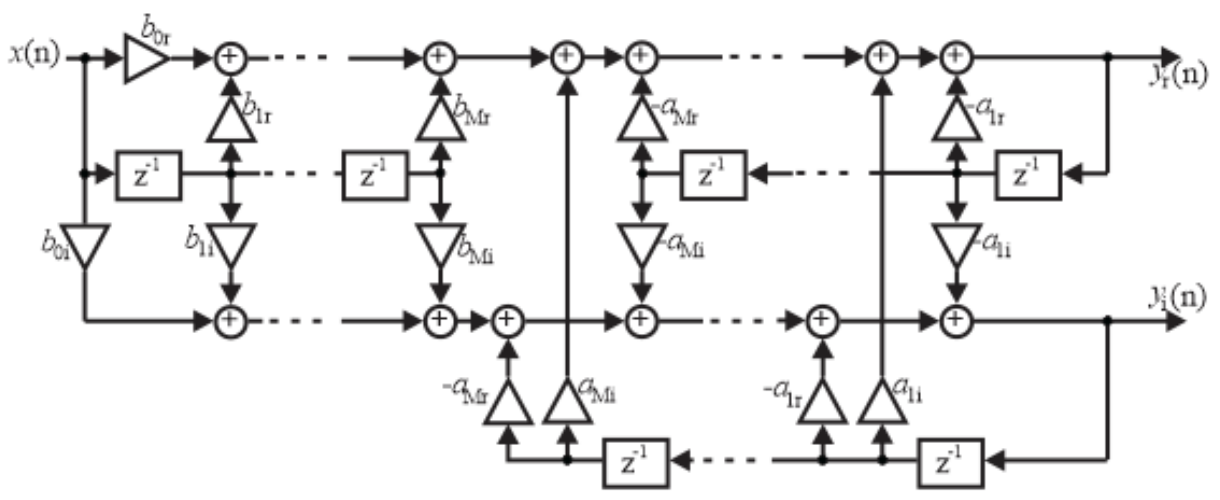

Fig. 6 Direct realization of digital filter with complex coefficients

\subsection{Canonic realization of digital filters with complex coefficients}

In order to get a canonic structure for realization transfer function of digital filter (5) must be expressed as a product of two complex functions:

$$
H(z)=H_{1}(z) H_{2}(z)=\frac{W(z)}{X(z)} \frac{Y(z)}{W(z)}
$$

where $W(z)=W_{r}+j W_{i}$.

Real and imaginary parts of the output can be obtained using the following expressions

$$
\begin{aligned}
y_{r}(n)= & b_{0 r} w_{r}(n)-b_{0 i} w_{i}(n)+b_{1 r} w_{r}(n-1)- \\
& b_{1 i} w_{i}(n-1)+b_{M r} w_{r}(n-M)-b_{M i} w_{i}(n-M)
\end{aligned}
$$

and 


$$
\begin{aligned}
y_{i}(n)= & b_{0 r} w_{i}(n)+b_{0 i} w_{r}(n)+b_{1 r} w_{i}(n-1)+ \\
& b_{1 i} w_{r}(n-1)+b_{M r} w_{i}(n-M)+b_{M i} w_{r}(n-M)
\end{aligned}
$$

where $w_{r}(n)$ and $w_{i}(n)$ are given with

$$
w_{r}(n)=x(n)-a_{1 r} w_{r}(n-1)+a_{1 i} w_{i}(n-1)-\ldots-a_{M r} w_{r}(n-M)+a_{M i} w_{i}(n-M)
$$

and

$$
w_{i}(n)=-a_{1 r} w_{i}(n-1)-a_{1 i} w_{r}(n-1)-\ldots-a_{M r} w_{i}(n-M)-a_{M i} w_{r}(n-M)
$$

From these expressions it is possible to calculate necessary hardware for realization of this structure. The number of required multipliers is $4 M+4$, adders $4 M+2$ and delay lines $2 \mathrm{M}$, but because of the canonic structure the number of required delay lines is minimal. Hence, total number of components for direct canonic realization is $8 M+4$ multipliers, $8 M+1$ adders and $2 M$ delay lines. The required number of components for filter of order $N / 2$ is $2 N+2$ multipliers, $2 N+1$ adders and $N$ delay lines. The direct canonic structure for realization of a digital filter with complex coefficients is displayed in Fig 7.

\subsection{Cascade realization of filter with complex coefficients}

Cascade realization is based on the breaking of the transfer function on the product of complex factors by the next manner

$$
H_{c}(z)=C \prod_{k=1}^{M} H_{c k}(z)
$$

where $C$ is a complex constant and $H_{c k}$ are complex sections of the first order, as opposed from realization of the real transfer functions where the second order sections exist. The complex section of the first order for cascade realization is given with

$$
H_{c 1}(z)=\frac{W(z)}{X(z)} \frac{Y(z)}{W(z)}=\frac{1+\left(b_{1 r}+j b_{1 i}\right) z^{-1}}{1+\left(a_{1 r}+j a_{1 i}\right) z^{-1}}
$$

where $W(z) / X(z)$ is defined as

$$
\frac{W(z)}{X(z)}=\frac{1}{1+\left(a_{1 r}+j a_{1 i}\right) z^{-1}}
$$

and

$$
\frac{Y(z)}{W(z)}=1+\left(b_{1 r}+j b_{1 i}\right) z^{-1}
$$

Applying an inverse $Z$ transformation after algebraic calculations real and imaginary output can be obtained as

$$
\begin{aligned}
& y_{r}(n)=w_{r}(n)+b_{1 r} w_{r}(n-1)-b_{1 i} w_{i}(n-1) \\
& y_{i}(n)=w_{i}(n)+b_{1 r} w_{i}(n-1)+b_{1 i} w_{r}(n-1)
\end{aligned}
$$

where $w_{r}(n)$ and $w_{i}(n)$ are given with 


$$
\begin{aligned}
& w_{r}(n)=x_{r}(n)-a_{1 r} w_{r}(n-1)+a_{1 i} w_{i}(n-1) \\
& w_{i}(n)=x_{i}(n)-a_{1 r} w_{r}(n-1)-a_{1 i} w_{r}(n-1)
\end{aligned}
$$

Using these expressions it is possible to calculate the number of components for realization of the complex section of the first order. The number of required multipliers is 8 , adders 8 and delay lines 2 . Complex section of the first order with a complex input is displayed in Fig. 8. Hence, the total number of components for cascade realization in this case is $8 M+2$ multipliers, $8 \mathrm{M}$ adders and $2 M$ delay lines. The required number of components for the filter of order $N / 2$ is $2 N+1$ multipliers, $2 N$ adders and $N / 2$ delay lines. For the case where the complex multiplier $C$ is the first component in the cascade structure all first order allpass sections have a complex input as displayed in Fig. 8.

If complex multiplier $C$ is not the first component in the cascade structure the first allpass section will have real input. In this case the structure for the first allpass section is displayed in Fig. 9.

It is recommended to put multiplier $C$ to be the first component in the cascade structure because in this case it requires a smaller number of components for realization comparing with the case when the first component in cascade is section of the first order with the real input. Section of the first order with the real input requires an adder less, but also two multipliers more due to the complex multiplier which is realized with two real multipliers.

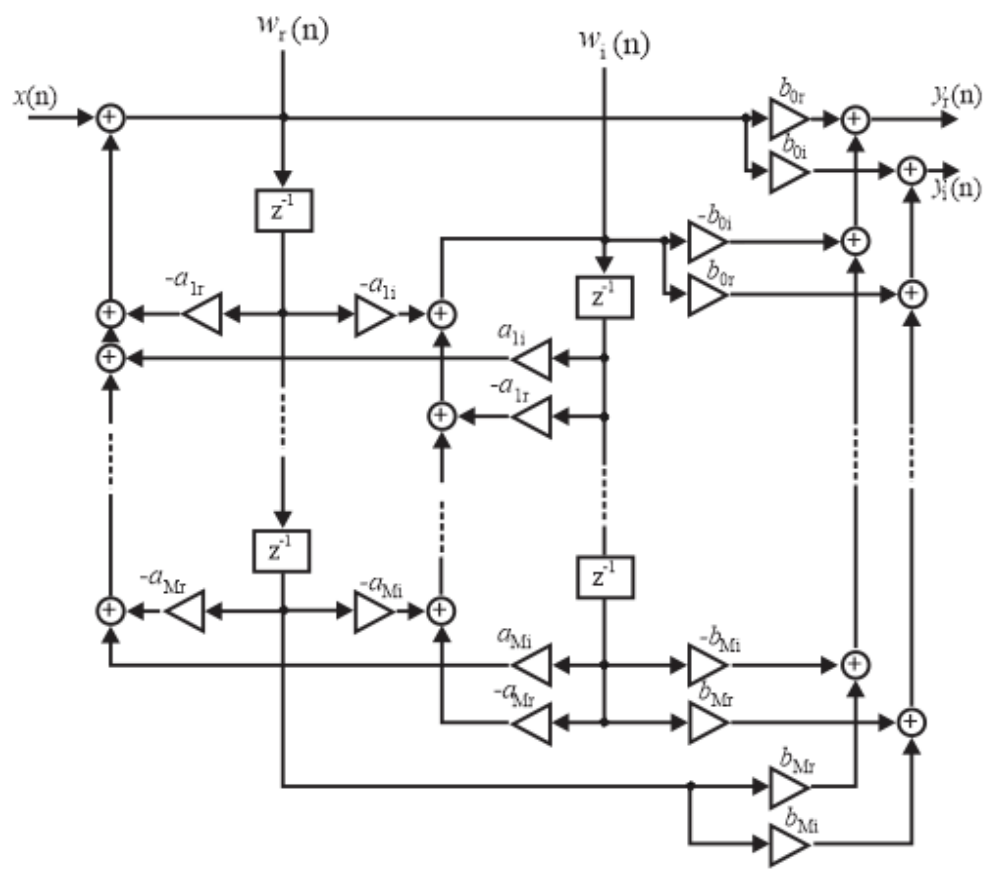

Fig. 7 Direct canonic realization of digital filter with complex coefficients. 


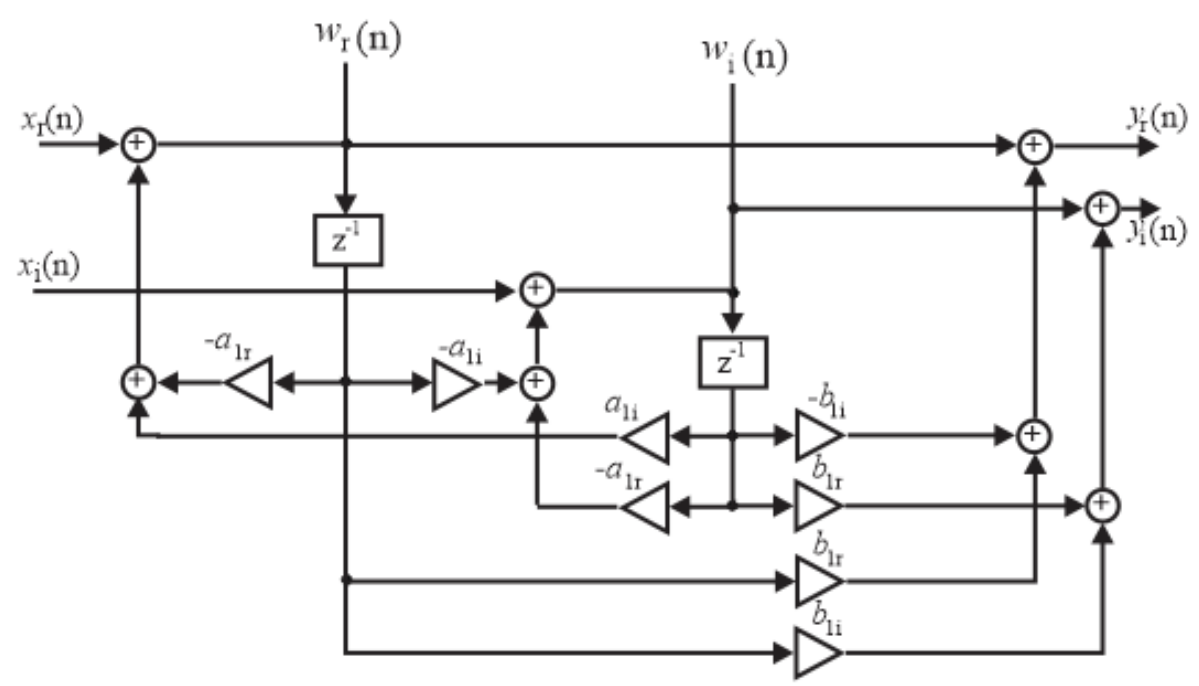

Fig. 8 Complex section of the first order for cascade realization

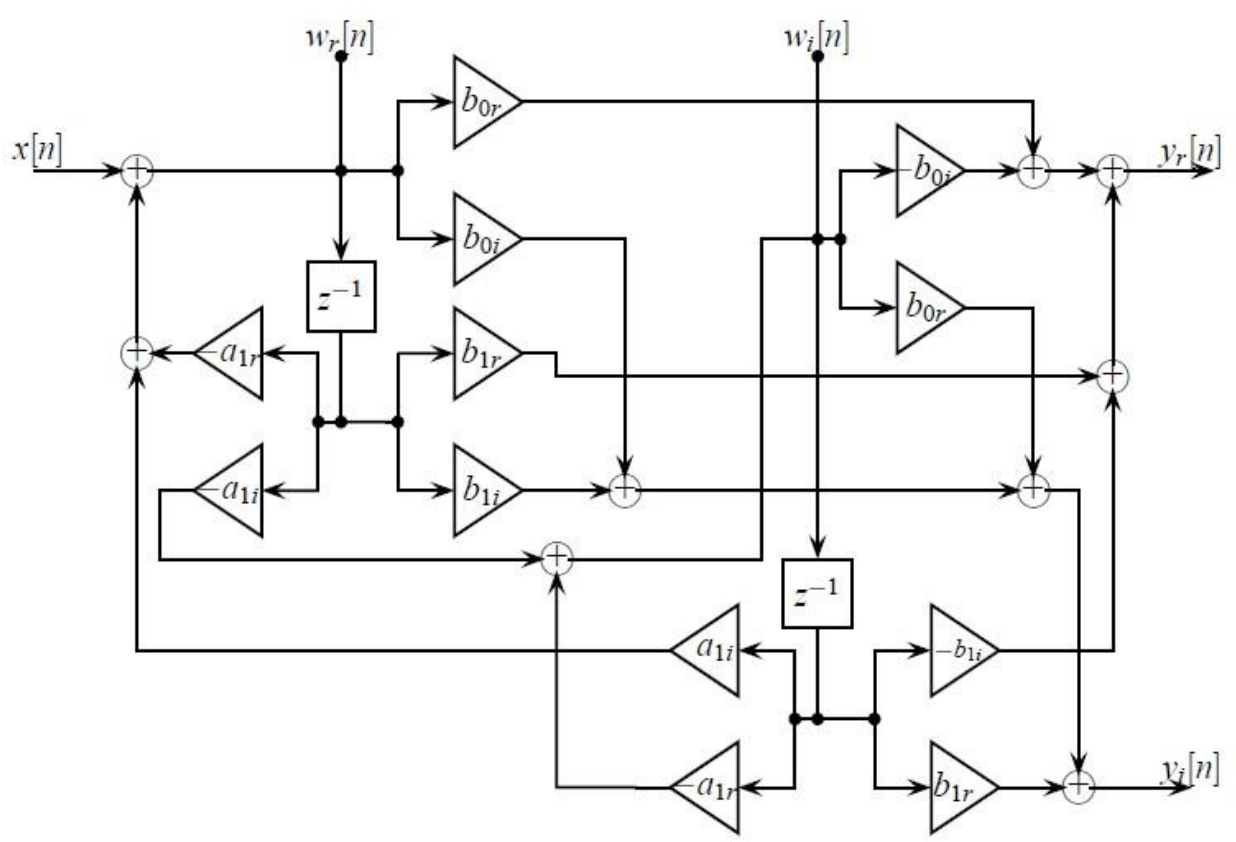

Fig. 9 The first allpass section for the case when multiplier C is not the first component in the cascade structure 


\subsection{Parallel realization of filter with complex coefficients}

A parallel realization is obtained by development of the transfer function into partial fractions:

$$
H_{p}(z)=C+\sum_{k=1}^{M} H_{p k}(z)
$$

Complex section of the first order for parallel realization is given with

$$
H_{1}(z)=\frac{b_{0 r}+j b_{0 i}}{1+\left(a_{1 r}+j a_{1 i}\right) z^{-1}}=\frac{W(z)}{X(z)} \frac{Y(z)}{W(z)}
$$

where $W(z)=W_{r}(z)+j W_{i}(z), Y(z)=Y_{r}(z)+j Y_{i}(z)$ and real input is $X(z)=X_{r}(z)$.

Applying an inverse $\mathrm{Z}$ transformation on $W(z) / X(z)$ we get

$$
\begin{aligned}
& y_{r}(n)=b_{0 r} w_{r}(n)-b_{0 i} w_{i}(n) \\
& y_{i}(n)=b_{0 r} w_{i}(n)+b_{0 i} w_{r}(n)
\end{aligned}
$$

where $w_{r}(n)$ and $w_{i}(n)$ are given with

$$
\begin{gathered}
w_{r}(n)=x(n)-a_{1 r} w_{r}(n-1)+a_{1 i} w_{i}(n-1) \\
w_{i}(n)=-a_{1 r} w_{i}(n-1)-a_{1 i} w_{r}(n-1)
\end{gathered}
$$

From equations for $w(n)$ and $y(n)$ the required hardware for realization of complex section of the first order for parallel structure can be obtained. The number of required multipliers is 8 , adders 5 and delay lines 2. Block schema of the complex function of the first order for parallel structure is displayed in Figure 10.

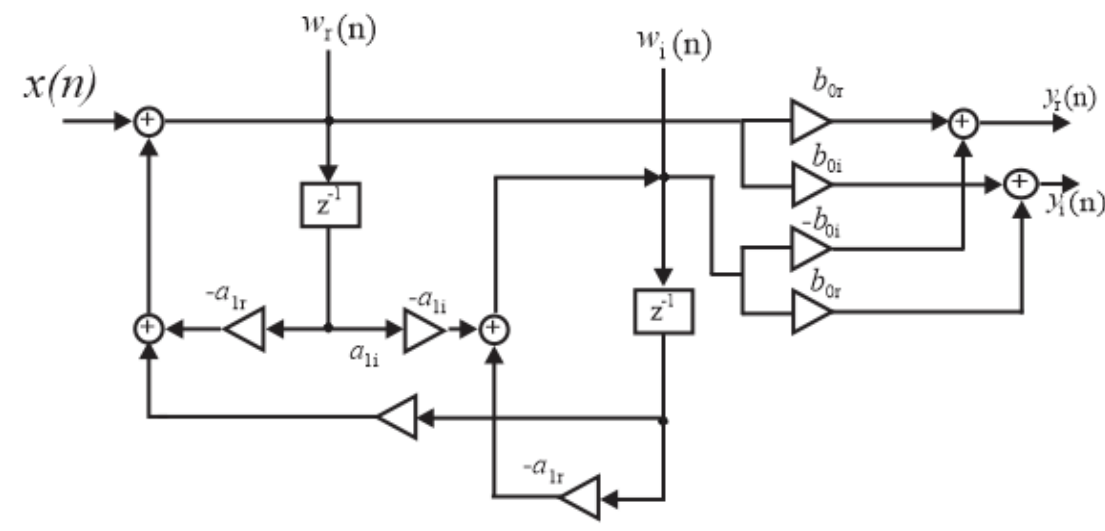

Fig. 10 Section of the first order for parallel realization of filter with complex coefficients. 
The total number of components for parallel realization of the digital filter with complex coefficients is multipliers $8 M+1$, adders $7 M$ and delay lines $2 M$. For a filter of order $N / 2$ total number of components is multipliers $(4 N+1) / 2$, adders $7 N / 4$ and delay lines $N / 2$.

The Table 3 shows the number of components necessary for realizing the transfer function using different structures.

Table 3 Hardware cost for filters with complex coefficients of order N/2.

\begin{tabular}{lccc}
\hline Structure & Delay lines & Multiplicators & Adders \\
\hline Direct realization & $3 \mathrm{~N} / 4$ & $3 \mathrm{~N} / 2+1$ & $3 \mathrm{~N} / 2$ \\
Canonic realization & $\mathrm{N} / 2$ & $2 \mathrm{~N}+2$ & $2 \mathrm{~N}+1$ \\
Cascade realization & $\mathrm{N} / 2$ & $2 \mathrm{~N}+1$ & $2 \mathrm{~N}$ \\
Parallel realization & $\mathrm{N} / 2$ & $(4 \mathrm{~N}+1) / 2$ & $7 \mathrm{~N} / 4$ \\
\hline
\end{tabular}

Comparing the number of delay lines for realization of one transfer function displayed in Table 1 and Table 3 we can conclude that for complex coefficients filter solution the necessary number of delay lines is lower. All structures described in this section correspond to arbitrary complex filters designed directly in the $\mathrm{z}$ domain. It is important to emphasize that the complex filters obtained by the allpass decomposition of even order real coefficients transfer function is a special case. Complex allpass filters possess coefficients in numerator and denominator which are conjugate complex to each other. In other words, instead of four, there are only two real coefficients. In that special case described configurations become even simpler and less hardware hungry. For example, the first order complex section in cascade structure requires 8 multipliers, but the complex allpass section needs only 4 multipliers.

\section{CONCLUSION}

Different structures for realization of digital filters with complex coefficients are investigated in this paper. Advantages for realization using the complex allpass sections are listed. In order to realize the complementary filters we presented connections between the maximal attenuation in the passband of lowpass filters and minimal attenuation in the passband of highpass filters. This connection must be satisfied in order to get usable complementary filters.

Classical structures for realization of complex coefficient filters obtained in the $\mathrm{z}$ domain are also presented. Calculation of hardware costs for all structures has been done. Analyzing hardware cost it is shown that the allpass filter realization requires fewer components. 


\section{REFERENCES}

[1] A. Fettweis: "Digital filter structures related to classical filter networks", Archiv Elek. Ubertranguns, Vol. 25, 1971, pp. 79-81.

[2] A. Fettweis: "Wave digital lattice filters", International Journal on Circuit Theory Applications, Vol. 2, 1974, pp. 203-211. https://doi.org/10.1002/CTA.4490020210

[3] J. Szczupak, S.K. Mitra: "On digital filter structures with low coefficient sensitivity", Proceedings IEEE, Vol. 66, 1978. DOI 10.1109/PROC.1978.11084

[4] P.P. Vaidyanathan, S.K. Mitra: "Passivity properties of low sensitivity digital filter structures", IEEE Transactions on Circuits and Systems, Vol. CAS-32(3), april, 1985, pp. 217-224. DOI 10.1109/ TCS.1985.1085693

[5] P.P. Vaidyanathan, P.A. Regalia, S.K. Mitra: "Design of doubly complementary IIR digital filters using a single complex allpass filters with multirate applications", IEEE Transactions on Circuits and Systems CAS-34, no. 4 (1987), pp. 378-389.

[6] P.P. Vaidyanathan, S.K. Mitra: "Very low sensitivity FIR filter implementation using 'structural passivity' concept", IEEE Transactions on Circuits and Systems, Vol. CAS-32, 1985, pp. 360-364. DOI 10.1109/TCS.1985.1085717

[7] P.P. Vaidyanathan, S.K. Mitra, Y. Neuvo: "A new approach to the realization of low sensitivity IIR digital filters", IEEE Transactions on Acoustic, Speech and Signal Processing, Vol. ASSP-34, 1986, pp. 350-361. DOI 10.1109/TASSP.1986.1164829

[8] P. DeWilde, E. Deprettere: "Orthogonal cascade realization of real multiport digital filters", International Journal on Circuits Theory Applications, Vol. 8, 1980, pp. 245-277.

[9] Iliev G., Nikolova Z, Poulkov V, Stoyanov G.: "Noise cancellation in OFDM systems using adaptive complex narrowband IIR filtering", IEEE International Conference on Communications ICC-2006, Istanbul Turkey, pp. 2859-2863. DOI 10.1109/ICC.2006.255214

[10] Iliev G., Nikolova Z, Stoyanov G., Egiazarian K.: "Efficient design of adaptive complex narrowband IIR filters", Proc. Of XII European Signal Proc. Conf. EUSIPCO 2004, Vienna, Austria, pp. 1597-1600. DOI 10.5281/zenodo. 38418

[11] Jiang H., Nishimura S., Hinamoto T.: "Steady-state analysis of complex adaptive IIR notch filter and its application to QPSK communication systems", IEICE Trans. Fundamentals, vol. E85-A, No. 5, May 2002, pp. 1088-1095. https://doi.org/10.1002/ecjc.1061

[12] Nikolova Z., Poulkov V., Iliev G., Egiazarian K.: "New adaptive complex IIR filters and their application in OFDM systems", Journal of Signal, Image and Video Processing, Springer, vol. 4, No. 2, June 2010, pp. 197-207, ISSN: 1863-1703. DOI 10.1007/s11760-009-0111-x

[13] Mitra, S. K. Digital Signal Processing, A Computer-Based Approach. McGraw-Hill, New York, 2006.

[14] Goran Stančić, Saša Nikolić. Digital linear phase notch filter design based on IIR all-pass filter application, ISSN 1051-2004, Digital Signal Processing, Vol. 23, No. 3, May 2013 , pp. 1065-1069. https://doi.org/10.1016/j.dsp.2013.01.006

[15] Saša Nikolić, Goran Stančić. "Design of IIR Notch Filter with Approximately Linear Phase", Circuits, Systems, and Signal Processing, Volume 31, Issue 6, 2012, pp 2119-2131, https://doi.org/10.1007/ s00034-012-9426-x

[16] Goran Stančić, Bojan Jovanović, Miljan Petrović. Complexity analysis of the quadratic phase IIR digital filters, Proceedings of $3^{\text {rd }}$ International Conference on Electrical, Electronic and Computing Engineering IcETRAN 2016, Zlatibor, Serbia, June 13-16, 2016, pp. ELI1.6. 1-5. 